INRA Prod. Anim., 2005, 18 (3), 203-208

\section{Besoins et apport en phosphore chez les poissons}

\author{
S.-J. KAUSHIK
}

UMR, NuAGe (Nutrition Aquaculture et Genomique), Station d'Hydrobiologie, F-64310 St-Pée-sur-Nivelle

Courriel : kaushik@st-pee.inra.fr
Constituant cellulaire et membranaire indispensable pour les réactions de phosphorylation, le phosphore $(\mathrm{P})$ joue un rôle de premier plan dans les métabolismes. L'importance du phosphore dans le développement du tissu osseux des poissons est bien établie. Les téléostéens (poissons osseux) ont des teneurs de plus de $15 \%$ dans les os. La chair de poissons est également riche en phosphore $(0,2$ à $0,8 \%)$. Parmi les éléments minéraux, le phosphore est celui dont le besoin est le plus important. Ainsi, l'étude du besoin nutritionnel en $P$ et sa disponibilité retiennent particulièrement l'attention des nutritionnistes. Les données actuelles montrent que, chez les différentes espèces de téléostéens et de crustacés, le besoin en phosphore disponible se situe entre 0,4 et 0,9\% de l'aliment. La disponibilité du $\mathrm{P}$ issu de différents ingrédients varie beaucoup entre espèces selon qu'elles aient ou non (agastres) un estomac.

\section{1 / Données sur le besoin en phosphore des poissons}

Malgré une forte interaction avec le milieu, les poissons ne peuvent couvrir leurs besoins uniquement par l'absorption branchiale ou cutanée. Même si une faible absorption du phosphore d'origine environnementale est démontrée chez le salmonidé, celle-ci ne peut pas couvrir les besoins. Le phosphore est, parmi tous les minéraux, celui pour lequel l'apport environnemental (exprimé en \% du besoin) est le plus faible. Un apport alimentaire est donc indispensable. Une carence en phosphore se traduit par l'anorexie, la diminution de la croissance squelettique et pondérale, la déminéralisation osseuse, des déformations squelettiques (Kaushik 1999, Lall 2002, Sugiura et al 2004). La minéralisation des structures osseuses (colonne vertébrale, opercules, écailles) est très dépendante de l'apport en phosphore. D'autres symptômes de carences sont un excès du dépôt lipidique et une augmentation de l'activité de certaines enzymes : phosphatase alcaline, enzymes impliquées dans la néoglucogénèse.

Les données actuelles disponibles pour différentes espèces de poissons téléostéens (nous ne disposons pas de données pour les poissons cartilagineux) montrent que le besoin en phosphore se situe entre 0,5 et $0,9 \%$ de l'aliment, constituant ainsi l'élément minéral quantitativement le plus important. Contrairement aux animaux terrestres, pour lesquels le rapport calcium/phosphore idéal a pu être bien établi, il n'en est pas de même chez les poissons. Ceci résulte d'une part des variations de la qualité de l'eau qui peuvent agir sur le besoin en calcium et d'autre part de la variabilité dans la disponibilité en phosphore contenu dans les matières premières. Chez les crevettes (Penaeus japonicus ou Penaeus vannamei), les besoins rapportés varient selon les auteurs de 0,7 à $1,2 \%$ de la ration avec une forte influence de l'apport relatif en Calcium (Ca) (Davis et Gatlin 1996). Ainsi, lorsque l'apport en Ca est faible, le besoin en $\mathrm{P}$ est de $0,35 \%$, et lorsque l'apport en Ca s'élève (1 à $2 \%)$, le besoin en $\mathrm{P}$ augmente et se situe à plus de $1 \%$ de la ration (tableau 1). Souvent, les données sont exprimées en \% de P total dans les aliments. Quelques travaux ont cependant pris en compte la disponibilité du P. Sur la base des données qui tiennent compte de la digestibilité, les besoins pour les poissons semblent se situer entre 0,5 à 0,6 \% de P disponible (Lall 2002). Chez la truite, le besoin en $\mathrm{P}$ disponible pour la croissance est de 0,37 et de 0,57\% pour une meilleure accrétion minérale. Les travaux de Rodehutscord et al (2000) montrent qu'à partir d'un apport de $0,4 \%$ de $\mathrm{P}$ disponible, l'excrétion de $\mathrm{P}$ augmente de façon linéaire chez la truite comme chez l'aiglefin (Roy et Lall 2003).

La méthode la plus couramment utilisée pour l'estimation du besoin, et qui a fait ses preuves, consiste à employer un aliment semi-purifié avec des apports variables en $\mathrm{P}$ sous forme de sels inorganiques, les réponses mesurées étant principalement la croissance pondérale accompagnée ou non d'autres critères (minéralisation osseuse, composition minérale, teneurs circulantes en minéraux, activités enzymatiques...). Une méthode plus récente, consiste à étudier l'excrétion des rejets métaboliques (Sugiura et al 2000), le niveau de besoin étant celui où l'on observe une augmentation de l'excrétion de $\mathrm{P}$ (figure 1).

Figure 1. Critères d'estimation du besoin en $P$ chez les poissons.

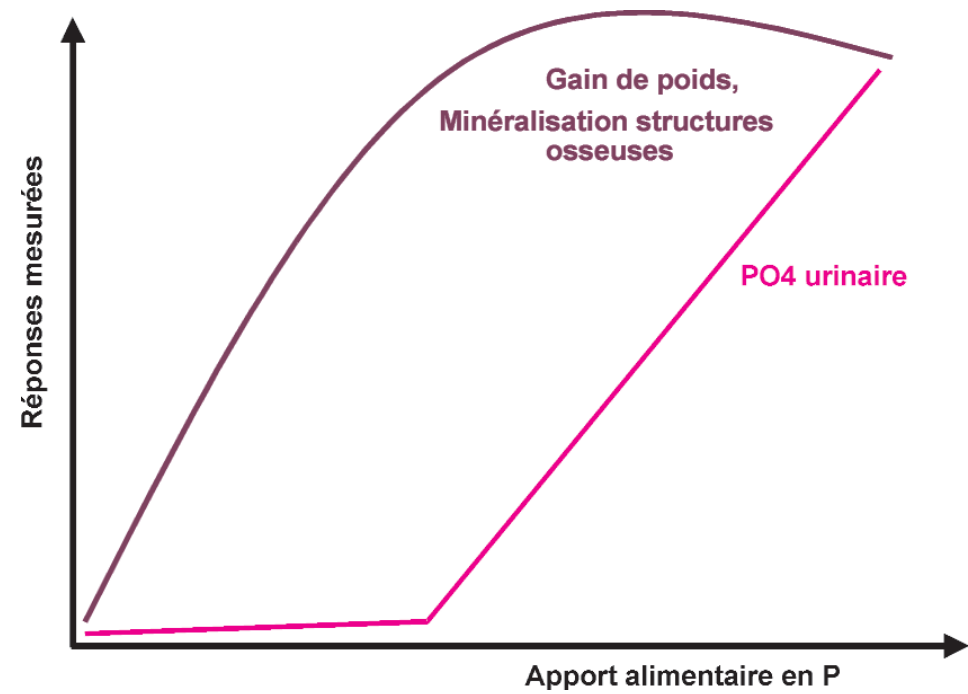


Tableau 1. Besoin en P (en g / kg de l'aliment) chez quelques poissons et crustacés d'élevage.

\begin{tabular}{|c|c|c|c|}
\hline \multicolumn{2}{|c|}{ Espèces } & \multirow{2}{*}{\begin{tabular}{|c|}
$\begin{array}{c}\text { Besoin } \\
\text { (g/ kg MS) }\end{array}$ \\
$6 ; 10-11$ \\
\end{tabular}} & \multirow{2}{*}{ 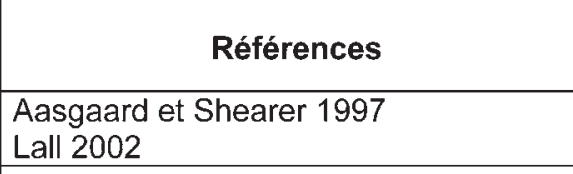 } \\
\hline Saumon Atlantique & Salmo salar & & \\
\hline Saumon du Pacifique & Oncorhynchus keta & 6 & Lall 2002 \\
\hline Truite arc-en-ciel & Oncorhynchus mykiss & 6 & Lall 2002 \\
\hline Poisson chat américain & Ictalurius punctatus & 4,5 & Lall 2002 \\
\hline Carpe commune & Cyprinus carpio & $6-7$ & Lall 2002 \\
\hline Carpe herbivore & Ctenopharyngodon idelle & 14 & Wang et al 2002 \\
\hline Carpe Amur & Mypharyngodom piceus & $4-6$ & Tang et Wang 1998 \\
\hline Carassin & Carassius carassius & $9-12$ & Tang et Wang 1998 \\
\hline Tilapia & Tilapia nilotica & $6-9$ & Lall 2002 \\
\hline Cichlidé d'Amérique & Cichlasoma urophthalmus & 15 & Chavez-Sanchez et al 2000 \\
\hline Mulet & Liza hematochiela & $6-7$ & El-Zibdeh et al 1995 \\
\hline Barbe de Sumatra & Barbus (Puntius) tetrazona & 5,2 & Elangovan et Shim 1998 \\
\hline Bar d'Amérique (Striped bass) & Morone saxatilis & 5,8 & Dougall et al 1996 \\
\hline Bar blanc (Hybrid striped bass) & $\begin{array}{l}\text { Morone chrysops (femelle) } \times M \text {. } \\
\text { saxatilis (male) }\end{array}$ & 6 & Brown et al 1993 \\
\hline Anguille japonaise & Anguilla japonicus & 3 & Lall 2002 \\
\hline Corégone ou Lavaret & Corégonus lavaretus & 7 & Vielma et al 2002 \\
\hline Perche (Silver perch) & Bidyanus bidyanus & 7 & Yang et al 2001 \\
\hline Ombrine & Sciaenops ocellatus & 9 & Davis et Robinson 1987 \\
\hline Daurade japonaise & Pagrus major & 7 & Lall 2002 \\
\hline Aiglefin & Melanogrammus aeglefinus & 10 & Roy et Lall 2003 \\
\hline Yellow croaker & Nibea albiflora & 5,8 & El-Zibdeh et al 1995 \\
\hline Sebaste (Rockfish) & Sebastes schlegeli & 3 & Lee et al 1998 \\
\hline Bar & Dicentrarchus labrax & 6,5 & Oliva-Teles et Pimentel-Rodrigues 2004 \\
\hline Milkfish & Chanos chanos & 8,5 & Borlongan et Satoh 2001 \\
\hline Black seabream & Sparus macrocephalus & 6,8 & Liu et al 1993 \\
\hline \multirow[t]{2}{*}{ Crevettes } & Peneaus japonicus & 10 à 20 & Davis et Gatlin 1996 \\
\hline & Peneaus vannamei & 3,5 à 20 & \\
\hline
\end{tabular}

\section{2 / Sources d'apport ali- mentaire et disponibilité}

Les farines de poisson qui constituent la principale source azotée des aliments des poissons en élevage intensif sont riches en phosphore, apporté essentiellement sous forme d'hydroxyapatite. La disponibilité du $P$ provenant de la farine de poisson est assez élevée chez la truite, le saumon ou le bar. Par contre, chez les cyprinidés, sa disponibilité est très faible (tableau 2). D’une façon générale, la disponibilité de $\mathrm{P}$ apporté sous forme inorganique est plus élevée que celle de P sous forme organique. Les sources inorganiques les plus solubles (phosphates mono ou bicalciques) ont en général une plus grande disponibilité que les sels moins solubles, tel que le phosphate tricalcique. Quant à la prévision du coefficient d'utilisation digestive apparent (CUDa) du phosphore, bien qu'il n'existe pas de méthodes fiables, une tentative récente de Hua et al (2005) mérite d'être citée. Les sources protéiques d'origine végétale sont riches en phosphore phytique avec une disponibilité de l'ordre de 40 à $60 \%$ chez les poissons. L'acide phytique (hexaphosphorique) peut se lier aux ions $\mathrm{Zn}^{2+}, \mathrm{Fe}^{2+}$ ou $\mathrm{Ca}^{2+}$, diminuant leur absorption intestinale et jouant ainsi le rôle de déminéralisant. Certes, on pourra améliorer sa disponibilité par l'ajout de phytases (d'origine microbienne par exemple) dans les aliments. Il semble que les crustacés soient capables d'utiliser le phosphore phytique grâce à une activité phytasique dans leur tube digestif.
Dans le contexte général de la substitution partielle ou totale des farines de poisson dans les aliments pour poissons, l'emploi d'ingrédients d'origine végétale induit une réduction de l'apport en $\mathrm{P}$ disponible, nécessitant une supplémentation adéquate pour couvrir les besoins. Il y a lieu aussi de prendre en compte des effets éventuels de l'acide phytique présent dans ces aliments. Comme chez les animaux terrestres, l'ajout de phytase permet d'améliorer l'utilisation digestive de $\mathrm{P}$ phytique tant chez les salmonidés (Rodehutscord et Pfeffer 1994, Cheng et Hardy 2003) que chez les poissons marins comme le bar (Oliva-Teles et al 1998). Une telle supplémentation permet aussi d'améliorer la disponibilité en d'autres minéraux $(\mathrm{Ca})$ ou oligo-éléments comme le magnésium, le manganèse et le zinc (Storebakken et al 1998). Ces auteurs 
Tableau 2. Digestibilité de $P(\%)$ de différentes sources chez quelques téléostéens.

\begin{tabular}{|l|c|c|c|c|}
\hline \multicolumn{1}{|c|}{ Sources } & Carpe & Truite & $\begin{array}{c}\text { Poisson } \\
\text { chat }\end{array}$ & Bar \\
\hline Inorganiques & 94 & 98 & 90 & - \\
\hline Mono phosphate de Na & 94 & 98 & - & - \\
\hline Mono phosphate de K & 94 & 94 & 94 & - \\
\hline Mono phosphate de Ca & 46 & 71 & 65 & - \\
\hline P dibasique de Ca & 13 & 64 & - & - \\
\hline P tribasique de Ca & 18 & $66-74$ & $39-40$ & $75-80$ \\
\hline Matières premières & - & 62 & - & - \\
\hline Farines de poisson & - & 64 & - & - \\
\hline Farine de plumes & 93 & 91 & - & - \\
\hline Farine sous produits volaille & - & 22 & $29-54$ & 36 \\
\hline Levure de bière & - & \multicolumn{5}{l|}{} \\
\hline Tourteau de soja & - & 9 & 25 & - \\
\hline Maïs & - & 75 & - & - \\
\hline Gluten de maïs & 57 & 58 & 28 & - \\
\hline Gluten de blé & - & 47 & - & - \\
\hline Germes de blé & $8-38$ & 19 & - & - \\
\hline Farine de blé
\end{tabular}

ont ainsi montré chez le saumon Atlantique qu'avec un aliment riche en concentré protéique de soja utilisé comme substitut à la farine de poisson, on observe une diminution sensible de la disponibilité en ces éléments, qui peut être rétablie par une supplémentation en phytase.

Le profil post-prandial d'excrétion du phosphate $\left(\mathrm{PO}_{4}\right)$ est assez semblable à celui qui est déjà décrit pour l'excrétion ammoniacale. Ainsi, après le repas, il y a une augmentation de l'élimination du $\mathrm{PO}_{4}$, la quantité totale excrétée étant liée à l'apport alimentaire en $\mathrm{P}$ disponible. Nous avons montré aussi que les profils d'excrétion restent semblables (figure 2) même avec des aliments ayant des caractéristiques très différen-

Figure 2. Profil d'excrétion post-prandiale du phosphate chez la truite recevant soit un aliment à fort taux de farine de poissons (FP) soit avec un concentré protéique de soja (CPS).

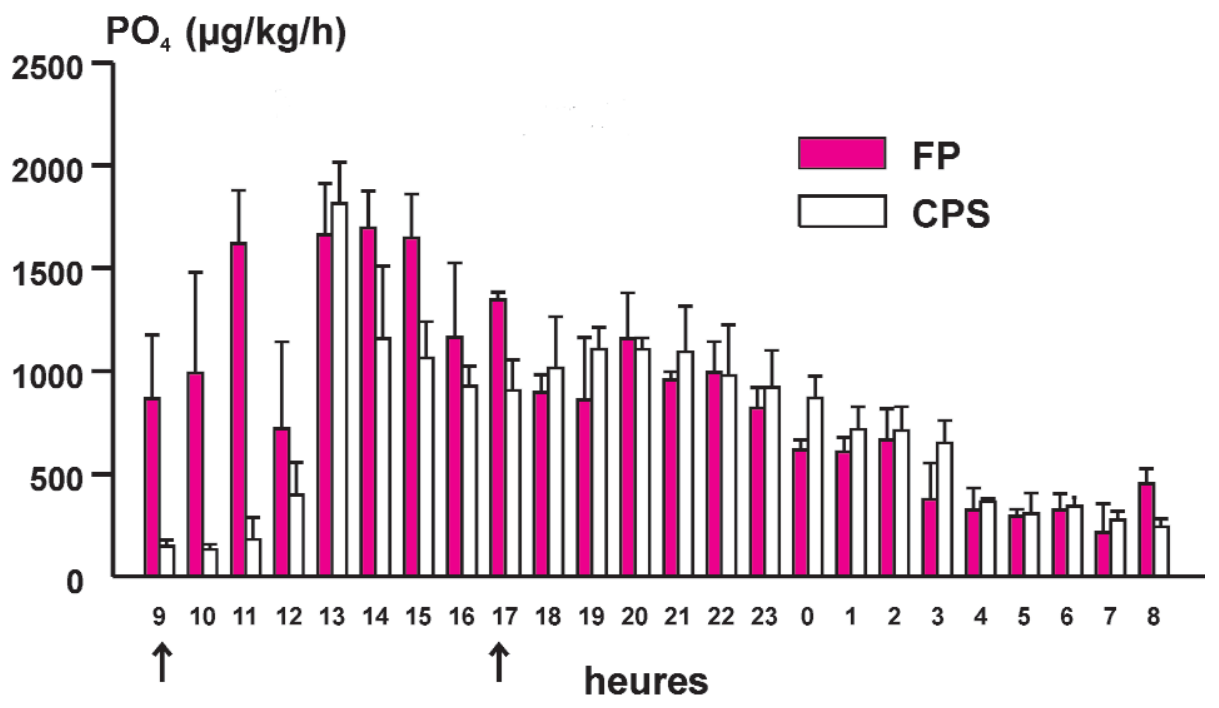

l'absorption intestinale du $\mathrm{P}$ chez les poissons. Sugiura et al (2003) suggèrent que l'absorption intestinale du $\mathrm{P}$ chez la truite serait également influencée par des facteurs environnementaux.

La chair des poissons est riche en phosphore, souvent > 250 mg / 100 g (à comparer avec le besoin journalier de l'homme qui est de l'ordre de $800 \mathrm{mg}$ ) et est aussi une source d'oligo-éléments peu présents dans l'alimentation humaine comme le zinc ou le sélénium. Pour une espèce donnée, la teneur en $\mathrm{P}$ du poisson entier est relativement stable. La teneur corporelle en $\mathrm{P}$ varie peu avec l'âge comme l'ont démontré Bureau et al (2003). Ils ont ainsi proposé pour la truite arc-en-ciel une teneur en $\mathrm{P}$ ( $\mathrm{g} /$ poisson) étant égale à $0.0036 \mathrm{x}$ poids vif (g) avec un $\mathrm{R}^{2}$ de 0,985 . Sur la base de nos données propres et de celles de la littérature, chez quelques espèces à intérêt aquacole, on peut retenir les valeurs suivantes pour la teneur en $\mathrm{P}$ du poisson entier ( $\mathrm{g} \mathrm{P} / \mathrm{kg}$ poids vif) : 4,4 à 4,8 pour le saumon Atlantique, 4,7 à 6 chez le bar et 6 à 6,7 chez la daurade. Même pour l'esturgeon, dont le squelette est cartilagineux, ces teneurs restent du même ordre : 4 à 6 g / kg poids frais. Avec un apport adéquat en $\mathrm{P}$ et en Ca dans les aliments, les structures osseuses (vertèbres) ont une teneur en $\mathrm{P}$ de l'ordre de 8 à $9 \%$ chez la truite arc-en-ciel et de 10 à $11 \%$ chez le saumon Atlantique. Skonberg et al (1997) ont montré que l'apport alimentaire en $\mathrm{P}$ a un effet plus important sur les teneurs en $\mathrm{P}$ de la peau (+ écailles) chez la truite arc-en-ciel, ce tissu cutané pouvant présenter des teneurs allant au delà de 10 g / kg poids frais. Selon ces auteurs, la teneur en $\mathrm{P}$ dans ce tissu est un excellent critère d'évaluation de l'état nutritionnel des poissons. Les écailles contiennent également de l'hydroxyapatite, un réservoir potentiel du $\mathrm{Ca}$ et du P. La résorption d'écailles, souvent constatée chez les poissons en fonction de stades physiologiques particuliers (migration anadrome, reproduction) permet sans doute ainsi de pallier un apport alimentaire faible.

Des travaux récents réalisés chez l'aiglefin (Roy et Lall 2003) ont montré qu'une augmentation de l'apport en phosphate monocalcique, entraîne une augmentation du nombre d'ostéoblastes et une diminution parallèle du nombre d'ostéoclastes. Compte tenu de l'importance des problèmes liés aux déformations squelettiques, rencontrés de plus en plus souvent chez les pois- 
Figure 3. Bilan simplifié de l'utilisation du P alimentaire chez le poisson.

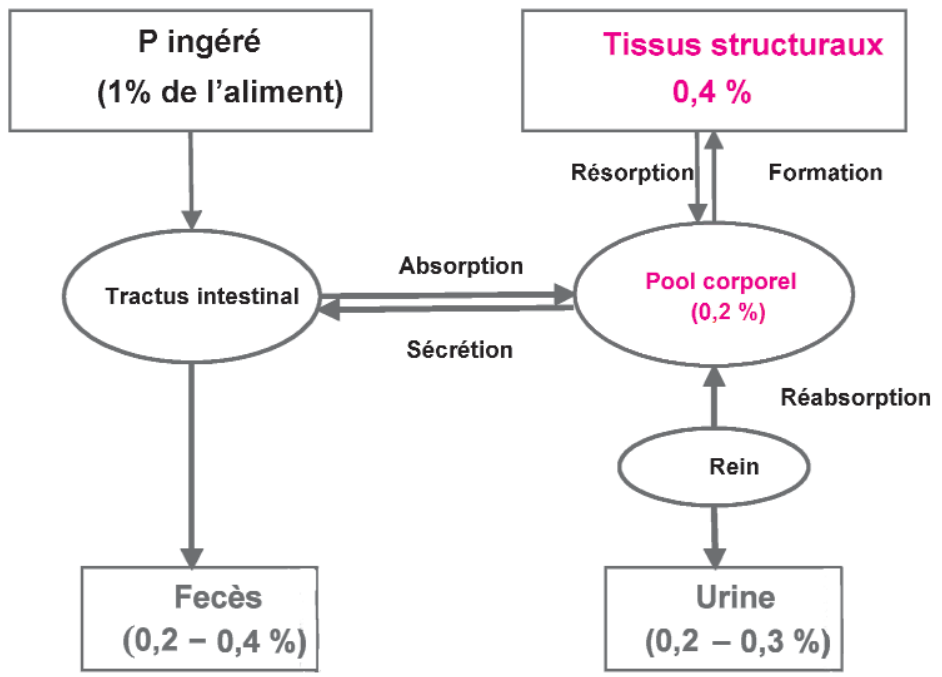

sons d'élevage, il y a lieu d'analyser de façon systématique l'équilibre entre la formation et la résorption osseuses et leur régulation par des facteurs nutritionnels à différents stades physiologiques.

\section{3 / Apport en phosphore et impacts environnementaux}

- Prise en compte des conséquences environnementales

Un des problèmes liés au phosphore en milieu aquatique est celui d'un apport optimal permettant de couvrir le besoin tout en évitant un excès de catabolites phosphorés. D'un point de vue de la qualité de l'eau, l'excès de phosphates se traduit souvent par une eutrophisation accrue des milieux aquatiques récepteurs. Bien que l'impact environnemental de l'aquaculture soit faible en termes de P comparé aux autres activités humaines et qu'une forte teneur en $\mathrm{PO}_{4}$ ne soit pas nuisible aux poissons au même titre que les rejets azotés sous forme ammoniacale, une meilleure utilisation du phosphore alimentaire par les poissons est nécessaire.

- Évolution des réglementations sur les rejets et sur la qualité des aliments

Dès 1989, la prise en compte des effets éventuels des rejets piscicoles dans le milieu aquatique a conduit à adopter des normes rigoureuses sur la quantité maximale d'apport en phosphore dans les aliments piscicoles. Certains pays comme le Danemark ont ainsi adopté des mesures limitant le taux du $\mathrm{P}$ dans les aliments pour poissons $(<1 \%)$. Dans d'autres pays, les réglementations portent plus sur une limitation de la production (par site, par bassin...) ou sur la qualité des effluents ( $N$, P, matière en suspension, demande biologique en oxygène). Une limitation de l'apport alimentaire admissible peut éventuellement conduire à une carence, si cet apport n'est pas sous une forme disponible pour les poissons. Compte tenu de l'importance que revêt un apport suffisant en phosphore biodisponible pour la croissance et le métabolisme, et compte tenu de l'amélioration de l'indice de consommation, il serait judicieux de raisonner en termes de besoin absolu (quantité nécessaire par unité de gain de poids) plus qu'en termes de concentration dans les aliments.

Quant aux stratégies pour une réduction des rejets en $\mathrm{P}$, les principes de base sont les suivants : emploi d'ingrédients à forte disponibilité en $\mathrm{P}$ permettant de couvrir les besoins des espèces concernées et éviter l'excès d'apport alimentaire. Il est clair que la diminution du taux d'incorporation de farines de poisson s'impose. Lors de l'emploi d'ingrédients d'origine végétale, si ce n'est que pour couvrir le besoin en $\mathrm{P}$, il faut vérifier que l'ajout de $\mathrm{P}$ inorganique est suffisant. Si l’apport végétal se traduit aussi par un apport en $\mathrm{P}$ phytique, l'emploi de phytase s'avère utile, surtout pour permettre une meilleure utilisation des oligo-éléments.

Une diminution de l'apport en $\mathrm{P}$ avec une meilleure disponibilité permet d'améliorer la rétention et de réduire les rejets en $\mathrm{P}$ (Robert et al 1993, Ketola et Richmond 1994). De nombreux travaux ont montré qu'il est possible de réduire les rejets en $\mathrm{P}$ par un remplacement même partiel des farines de poisson par des ingrédients végétaux (oléagineux, protéagineux, céréales), chez la truite arc-en-ciel mais également chez d'autres espèces. Chez la carpe, même avec un apport alimentaire en $\mathrm{P}$ de 1 à $1,4 \%$ de l'aliment, une substitution partielle de la farine de poisson par un mélange d'autres sources protéiques d'origine animale ou végétale (farine de plumes, farine de sang, tourteau de soja) permet de réduire les rejets en phosphore (Jahan et al 2001). Chez le bar, le remplacement quasi total de la farine de poisson permet ainsi de réduire de façon très significative les rejets (Kaushik et al 2004). Pour le calcul des rejets en $P$, un modèle est également proposé, validé par ailleurs dans des conditions de trutticulture française (Papatryphon et al 2005).

\section{Conclusion}

Les données actuelles sur les besoins en phosphore chez les poissons et les crustacés montrent une certaine variabilité, se situant entre 0,4 et $0,9 \%$ de l'aliment. Dans le contexte de l'élevage intensif, si la substitution des farines de poisson par d'autres ingrédients d'origine végétale permet de réduire l'excès d'apport en phosphore et ainsi l'impact environnemental, il y a lieu de bien s'assurer de la disponibilité du phosphore qui varie beaucoup entre ingrédients et entre espèces. Une meilleure connaissance des mécanismes d'absorption du phosphore et de son implication dans le métabolisme des tissus structuraux permettra d'assurer à la fois la qualité de l'environnement et celle des produits. 


\section{Références}

Aasgaard T., Shearer K.D., 1997. Dietary phosphorus requirement of juvenile Atlantic salmon, Salmo salar L.. Aquacult. Nutr., 3, 1723.

Borlongan I.G., Satoh S., 2001. Dietary phosphorus requirement of juvenile milkfish, Chanos chanos (Forsskal). Aquacult. Res., 32, 26-32.

Brown M.L., Jaramillo F. Jr., Gatlin D.I., 1993. Dietary phosphorus requirement of juvenile sunshine bass, Morone chrysops $\mathrm{x}$ M. saxatilis. Aquaculture, 113, 355-363.

Bureau D.P., Gunther S.J., Cho C.Y., 2003. Chemical composition and preliminary theoretical estimates of waste outputs of rainbow trout reared in commercial cage culture operations in Ontario. N. Am. J. Aquacult., 65, 33-38.

Chavez-Sanchez C., Martinez-Palacios C.A., Martinez-Perez G., Ross L.G., 2000. Phosphorus and calcium requirements in the diet of the American cichlid Cichlasoma urophthalmus (Guenther). Aquacult. Nutr., 6, 1-9.

Cheng Z.J., Hardy R.W., 2003. Effects of extrusion and expelling processing, and microbial phytase supplementation on apparent digestibility coefficients of nutrients in full-fat soybeans for rainbow trout (Oncorhynchus mykiss). Aquaculture, 218, 501-514.

Davis D.A., Gatlin D.M., 1996. Dietary mineral requirements of fish and marine crustaceans. Rev. Fish. Sci., 4, 75-99.

Davis D.A., Robinson E.H., 1987. Dietary phosphorus requirement of juvenile red drum Sciaenops ocellatus. J. World Aquacult. Soc., 18, 129-136.

Dougall D.S., Woods L.C., III, Douglass L.W., Soares J.H., 1996. Dietary phosphorus requirement of juvenile striped bass Morone saxatilis. J. World Aquacult. Soc., 27, 82-91.

El-Zibdeh M., Ide K., Yoshimatsu T., Matsui S., Furuichi M., 1995. Requirement of yellow croaker Nibea albiflora for dietary phosphorus. J. Fac. Agric. Kyushu Univ., 40, 147-155.

Elangovan A. Shim K.F., 1998. Dietary phosphorus requirement of juvenile tiger barb, Barbus tetrazona (Bleeker 1855). Aquarium Sci. Conserv., 2, 9-19.

Hua K., Liu L., Bureau D.P., 2005. Determination of phosphorus fractions in animal protein ingredients. J. Agri Food Chem., 53, 1571-1574.

Jahan P., Watanabe T., Satoh, S., Kiron, V., 2001. Formulation of low phosphorus loading diets for carp (Cyprinus carpio L.). Aquacult. Res., 32, 361-368.

Kaushik S., 1999. Nutrition minérale. In : Nutrition et alimentation des poissons et crustacés, J. Guillaume, S. Kaushik, P. Bergot, R. Métailler, (Eds), INRA Editions, 213-227.
Kaushik S. J., Coves D., Dutto G., Blanc D., 2004. Almost total replacement of fish meal by plant protein sources in the diet of a marine teleost, the European seabass, Dicentrarchus labrax. Aquaculture, 230, 391-404.

Ketola H.G., Richmond M.E., 1994. Requirement of rainbow trout for dietary phosphorus and its relationship to the amount discharged in hatchery effluents. Trans. Am. Fish. Soc., 123, 587-594.

Lall S.P., 2002. The Minerals. Fish Nutrition, Third Edition. R. H. Hardy, Halver, J.E. (Eds), Academic Press, Elsevier Science, USA, 259308.

Lee S.M., Park S.R., Kim J.D., 1998. Dietary optimum phosphorus level of juvenile Korean rockfish (Sebastes schlegeli). J. Fish. Sci. Tech., 1, 180-186.

Liu J., Maotang L., Keling W., Xincheng W., Jiankang L., 1993. Studies on the phosphorus requirement and proper calcium/phosphorus ratio in the diet of the black sea bream (Sparus macrocephalus). Proc. Int. Conf, World Aquaculture Society, Torremolinos, Spain, 406.

Médale F., Boujard T., Vallee F., Blanc D., Mambrini M., Roem A., Kaushik S.J., 1998. Voluntary feed intake, nitrogen and phosphorus losses in rainbow trout (Oncorhynchus mykiss) fed increasing dietary levels of soy protein concentrate. Aquat. Living Resour., 11, 239-246.

Oliva-Teles A., Pimentel-Rodrigues A., 2004. Phosphorus requirement of European sea bass (Dicentrarchus labrax L.) juveniles. Aquacult. Res., 35, 636-642.

Oliva-Teles A., Pereira J.P., Gounveia A., Gomes E., 1998. Utilisation of diets supplemented with microbial phytase by seabass (Dicentrachus labrax) juveniles. Aquat. Living Resour., 11, 255-259.

Papatryphon E., Petit J., Hayo V., Kaushik S.J., Claver K., 2005. Nutrient balance modeling as a tool for environmental management in aquaculture: The case of trout farming in France. Env. Managem., 35, 161-174.

Robert N., Le Gouvello R., Mauviot J.C., Arroyo F., Aguirre P., Kaushik S.J., 1993. Use of extruded diets in intensive trout culture: Effects of protein to energy ratios on growth, nutrient utilization and on flesh and water quality. In: Fish Nutrition in practice, S.J. Kaushik, P. Luquet (Eds), Colloques de l'INRA, 61, Paris, France, 497-500.

Rodehutscord M., Pfeffer E, 1994. The effect of supplemental microbial phytase on P digestibility and utilization in rainbow trout (Oncorhynchus mykiss). Water Sci. Tech., 31, 143-148.

Rodehutscord M., Gregus Z., Pfeffer E., 2000. Effect of phosphorus intake on faecal and nonfaecal phosphorus excretion in rainbow trout
(Oncorhynchus mykiss) and the consequences for comparative phosphorus availability studies. Aquaculture, 188, 383-398.

Roy P.K., Lall S.P., 2003. Dietary phosphorus requirement of juvenile haddock (Melanogrammus aeglefinus L.). Aquaculture, 221, 451468.

Satoh S., Hernandez A., Tokoro T., Morishita Y., Kiron,V., Watanabe T., 2003. Comparison of phosphorus retention efficiency between rainbow trout (Oncorhynchus mykiss) fed a commercial diet and a low fish meal based diet. Aquaculture, 224, 271-282.

Skonberg D.I., Yogev L., Hardy R.W., Dong F.M., 1997. Metabolic response to dietary phosphorus intake in rainbow trout (Oncorhynchus mykiss). Aquaculture, 157, $11-24$

Storebakken T., Shearer K.D., Roem A.J., 1998. Availability of protein, phosphorus and other elements in fish meal, soy-protein concentrate and phytase-treated soy-protein-concentrate-based diets to Atlantic salmon, Salmo salar. Aquaculture, 16, 365-379.

Sugiura S.H., Dong F.M., Hardy R.W., 2000. A new approach to estimating the minimum dietary requirement of phosphorus for large rainbow trout based on nonfecal excretions of phosphorus and nitrogen. J. Nutr., 130, 865-872.

Sugiura S.H., McDaniel N.K., Ferraris R.P., 2003. In vivo fractional $\mathrm{Pi}$ absorption and $\mathrm{NaPi}-$ II mRNA expression in rainbow trout are upregulated by dietary P restriction. Am. J. Physiol. Regul. Integr. Comp. Physiol., 285, R770-R781.

Sugiura S.H., Hardy R.W., Roberts R.J., 2004. The pathology of phosphorus deficiency in fish a review. J. Fish Diseases, 27, 255-265.

Tang Z., Wang D., 1998. Dietary calcium and phosphorus requirements of black carp and allogenetic crucian carp. J. Shanghai Fish. Univ., 7, suppl., 140-147.

Vielma J., Koskela J., Ruohonen K., 2002. Growth, bone mineralization, and heat and low oxygen tolerance in European whitefish (Coregonus lavaretus L.) fed with graded levels of phosphorus. Aquaculture, 212, 321-333.

Yang S.D., Lin T. S., Liou C.H., Peng H.K., 2001. Dietary phosphorus requirement of silve perch (Bidyanus bidyanus). $6^{\text {th }}$ Asian Fisheries Forum Book of Abstracts, 274.

Wang Z., Sun H., Liu H., Ma J., Xuan Z., Ren, W., Wu, Y., 2002. The requirements and optimum ratio of calcium and phosphorus in the compound feed of Ctenopharyngodon idellus. J. Fisheries of China/Shuichan Xuebao, 26, 127 132. 


\title{
Résumé
}

Quantitativement, dans l'alimentation des poissons, le phosphore est l'élément minéral le plus important. Les données actuelles montrent que, chez les différentes espèces de téléostéens et de crustacés, le besoin en phosphore disponible se situe entre 0,4 et $0,9 \%$ de l'aliment. La disponibilité du phosphore issu de différents ingrédients varie beaucoup entre espèces. Une fois le besoin couvert, l'excès de phosphore semble s'éliminer par voie urinaire. Nos connaissances sur le flux de phosphore chez les poissons sont cependant assez limitées. Il y a lieu aussi d'acquérir des données sur le rôle du phosphore d'origine alimentaire dans la régulation de l'état dynamique des structures osseuses et dans la prévention des déformations squelettiques. Du point de vue du maintien de la qualité du milieu, l'excès de phosphore se traduit souvent par une eutrophisation accrue des milieux aquatiques récepteurs. Même si l'impact environnemental de l'aquaculture demeure faible en termes de phosphore comparé aux autres activités humaines et qu'une forte teneur en phosphates ne soit pas nuisible aux poissons, il y a lieu d'éviter un apport en excès et d'assurer la meilleure utilisation des apports. Compte tenu du développement de l'aquaculture, de la diversité des espèces rencontrées et des enjeux liés à la diminution de la farine de poissons dans leur alimentation, l'étude du besoin nutritionnel en $\mathbf{P}$ et sa disponibilité retiennent une attention particulière des nutritionnistes.

\begin{abstract}
Phosphorus needs and intake in fish

Quantitatively, phosphorous is the major mineral in fish nutrition. Available data for different species of teleosts or crustaceans show that dietary P supply should be between 0.4 and $0.9 \%$ of the diet. The availability of phosphorus from feed ingredients varies between species, depending upon the presence or absence of gastric digestion. Once the requirements are satisfied, a major portion of absorbed $P$ is released through urinary excretion. Our current knowledge on phosphorus flow in fish is rather limited. There is also a need for a greater understanding of the role of dietary phosphorus in the regulation of formation and resorption of skeletal tissues. From the environmental point of view, given the potential adverse effects of excess $P$ in promoting eutrophication of water bodies, there is more than ever, a serious need for optimising dietary supply, improving availability and decreasing excess effluent discharge into the aquatic environment.
\end{abstract}

KAUSHIK S.-J., 2005. Besoins et apport en phosphore chez le poisson. INRA Prod. Anim., 18, 203-208. 